\title{
Breast cancer cell cyclooxygenase-2 expression alters extracellular matrix structure and function and numbers of cancer associated fibroblasts
}

\author{
Balaji Krishnamachary ${ }^{1, *}$, Ioannis Stasinopoulos ${ }^{1, *}$, Samata Kakkad ${ }^{1}$, Marie-France \\ Penet $^{1,2}$, Desmond Jacob ${ }^{1}$, Flonne Wildes ${ }^{1}$, Yelena Mironchik ${ }^{1}$, Arvind P. Pathak ${ }^{1,2}$, \\ Meiyappan Solaiyappan ${ }^{1}$, Zaver M. Bhujwalla ${ }^{1,2}$ \\ ${ }^{1} \mathrm{JHU}$ ICMIC Program, Division of Cancer Imaging Research, The Russell H. Morgan Department of Radiology and Radiological \\ Science, Baltimore, MD 21205, USA \\ ${ }^{2}$ Sidney Kimmel Comprehensive Cancer Center, The Johns Hopkins University School of Medicine, Baltimore, MD 21205, USA \\ *These authors have contributed equally to this work \\ Correspondence to: Zaver M. Bhujwalla, email: zaver@mri.jhu.edu \\ Keywords: COX-2, cancer associated fibroblasts, collagen 1 fibers, macromolecular transport, metastasis \\ Received: August 04, $2016 \quad$ Accepted: December 27, 2016 Published: January 31, 2017
}

\section{ABSTRACT}

Cyclooxygenase-2 (COX-2) is a critically important mediator of inflammation that significantly influences tumor angiogenesis, invasion, and metastasis. We investigated the role of COX-2 expressed by triple negative breast cancer cells in altering the structure and function of the extracellular matrix (ECM). COX-2 downregulation effects on ECM structure and function were investigated using magnetic resonance imaging (MRI) and second harmonic generation (SHG) microscopy of tumors derived from triple negative MDA-MB-231 breast cancer cells, and a derived clone stably expressing a short hairpin (shRNA) molecule downregulating COX-2. MRI of albumin-GdDTPA was used to characterize macromolecular fluid transport in vivo and SHG microscopy was used to quantify collagen 1 (Col1) fiber morphology. COX-2 downregulation decreased Col1 fiber density and altered macromolecular fluid transport. Immunohistochemistry identified significantly fewer activated cancer associated fibroblasts (CAFs) in low COX2 expressing tumors. Metastatic lung nodules established by $\mathrm{COX}-2$ downregulated cells were infrequent, smaller, and contained fewer Col1 fibers.

COX-2 overexpression studies were performed with tumors derived from triple negative SUM-149 breast cancer cells lentivirally transduced to overexpress COX-2. SHG microscopy identified significantly higher Col1 fiber density in COX-2 overexpressing tumors with an increase of CAFs. These data expand upon the roles of COX-2 in shaping the structure and function of the ECM in primary and metastatic tumors, and identify the potential role of COX-2 in modifying the number of CAFs in tumors that may have contributed to the altered ECM.

\section{INTRODUCTION}

Cyclooxygenase-2 (COX-2) is an active mediator of the inflammatory response of cells [1]. Its major role in a multitude of degenerative diseases such as autoimmune diseases [2], gastric inflammation [3], and several cancers, such as gastric [4], lung [5], breast [6, 7] and colon cancer [8], has resulted in the development of pharmaceutical inhibitors targeting COX-2. However the side effects of these agents $[9,10]$ have diminished the prospects of their use in cancer treatment. Nevertheless, COX-2 remains one of the most important targets in cancer, especially for cancers that are COX-2-dependent [7, 11-13]. The promise of molecular agents such as small interfering RNA (siRNA), that are more specific than pharmacological interventions, provides renewed hope for exploiting this target [14].

In breast cancer, several studies have highlighted the importance of COX-2 in tumor development, progression, invasion, and metastasis [15-17]. Uncovering mechanisms by which $\mathrm{COX}-2$ regulates these processes can provide new insights and identify novel targets. In a study of 
127 patients, triple negative breast cancer (TNBC) was found to be an independent predictor for $\mathrm{COX}-2$ overexpression [18]. Silencing COX-2 in MDA-MB-231 metastatic TNBC cells inhibited tumor onset and growth in an orthotopic xenograft model, and inhibited pulmonary colonization in an experimental model of metastasis [17]. These changes were attributed to reduced invasiveness, reduced angiogenic capabilities, and reduced expression of pro-metastatic components of the extracellular matrix (ECM) [19]. COX-2 inhibition has been found to significantly reduce the expression of degradative enzymes such as matrix metalloproteinase 1 (MMP1), and alter the expression of ECM components such as hyaluronan and lumican that play a role in intra-fibrillar collagen spacing $[17,19]$. The role of prostaglandins produced by COX-2 in promoting cancer cell adhesion in the ECM has been extensively reviewed [20].

More recently, COX-2 has been investigated within the context of ECM modification. Pharmacological inhibition of COX-2 was found to reduce collagen deposition and tumor growth in the MMTV-PyMT or MMTV-PyMT/Colla1 mouse models [21], and invasion during mammary gland involution $[21,22]$. The alignment of collagen fibers perpendicularly to the tumor boundary was also associated with decreased disease-free survival in breast cancer patients [22]. The role of COX-2 mediated collagen deposition and remodeling in breast cancer metastasis is also being actively investigated [21, 23]. A high density of collagen 1 (Col1) fibers in the tumor ECM has been identified as a predictor of increased metastasis $[24,25]$.

Here we investigated the role of $\mathrm{COX}-2$ expression by TNBC cells in shaping the structure and function of the tumor ECM. Studies were performed in triple negative MDA-MB-231 tumors derived from cells with COX2 downregulated by stable expression of COX-2 short hairpin RNA (shRNA) and in triple negative SUM-149 tumors derived from cells with COX-2 overexpressed following lentiviral transduction. These tumors were used to investigate the relationship between COX-2 expression, vascular parameters, and macromolecular transport, using MRI, and Col1 fiber distribution, using second harmonic generation (SHG) confocal microscopy. We investigated the ability of these cells to spontaneously metastasize to the lymph nodes and to establish metastatic nodules in lungs in an experimental model of metastasis. Coll fiber patterns in the lung nodules were characterized. While cancer cells shape Coll fiber patterns through the secretion of various enzymes [26], Coll fiber is primarily synthesized by activated cancer associated fibroblasts (CAFs) within the tumor [27]. We therefore quantified the number of activated CAFs in the tumors using immunohistochemistry and immunoblotting for alphasmooth muscle actin ( $\alpha$-SMA).

We identified significant differences in vascular permeability and macromolecular transport in COX-2 downregulated MDA-MB-231 tumors together with a significant decrease of vascular endothelial growth factor (VEGF) that explained the decrease of vascular permeability detected with MRI. Sparser Coll fibers were evident with COX-2 downregulation in primary tumors together with fewer and smaller metastatic nodules. Both primary tumors and metastatic nodules contained fewer CAFs.

COX-2 overexpressing SUM-149 tumors displayed increased Coll fiber density with a higher number of CAFs. These data expand upon the role of COX-2 in modifying the structure and function of the ECM, and identify the potential role of COX-2 in activating fibroblasts in the tumor.

\section{RESULTS}

COX-2 levels were significantly lower in MDA-MB-231 Clone 13 cells; these cells could only be moderately induced to express COX-2 with 12-O-tetradecanoylphorbol-13-acetate (TPA) (Figure 1A), and secrete the COX-2 product prostaglandin $\mathrm{E}_{2}\left(\mathrm{PGE}_{2}\right)$ following induction with TPA (Figure 1B). Injection of COX-2-reduced Clone 13 cells with Matrigel in the mammary fat pad gave rise to tumors with significantly delayed onset as shown in growth curves (Figure 1C) that correlated well with shRNA-mediated reduction of COX2 and COX-2-catalyzed $\mathrm{PGE}_{2}$ formation. The decrease in COX-2 expression in tumors derived from Clone 13 cells were confirmed from protein expression (Figure 1D) and mRNA levels (Figure 1E).

To evaluate functional changes in the ECM we injected the macromolecular contrast agent albuminGdDTPA $(\sim 100 \mathrm{kDa})$ i.v. and followed its in vivo distribution noninvasively in volume-matched tumors. This allowed us to derive macromolecular transport parameters as well as evaluate the permeability of the tumor vasculature to this contrast agent. Representative MR derived images of permeability (Figure 2A, top), influx rate (Figure 2A, middle) and efflux rate (Figure 2A, bottom) show the effect of COX-2 reduction on permeability and macromolecular transport. Quantification of these parameters is shown in Figure 2B for permeability (top), influx rate (middle) and efflux rate (bottom). Permeability and macromolecular transport were significantly lower in COX-2 downregulated Clone 13 tumors. The scale in the efflux rate panel is inverted with cooler colors reflecting faster draining of the contrast agent. A significant decrease of VEGF protein (Figure 2C) and mRNA (Figure 2D) was observed in Clone 13 tumors.

To evaluate the effect of COX-2 expression on structural ECM changes, we characterized Coll fiber distribution in $1 \mathrm{~mm}$-thick fresh tumor slices using second harmonic generation (SHG) microscopy. Representative images of Coll fibers from a z-stack are displayed in Figure 3A that demonstrate the reduced Coll fiber content in Clone 13 tumors compared to MDA-MB-231 tumors. 
Clone 13 tumors with COX-2 downregulated contained fewer Col1 fibers with significantly increased mean interfiber distance (Figure 3B, left) and reduced fractional fiber volume (Figure 3B, right).

COX-2 downregulation in MDA-MB-231 cells resulted in fewer and smaller metastatic lung nodules in an experimental model of metastasis. Representative hematoxylin and eosin (H\&E) stained lung sections, shown in Figure 4A, demonstrate the reduction in colonization and establishment of pulmonary metastasis following COX-2 downregulation. Figure 4B shows the significant decrease of metastatic burden observed following COX-2 downregulation.

Metastatic lung nodules established by Clone 13 cells had fewer Coll fibers in the nodules compared to nodules established by COX-2 expressing MDA-MB-231 cells (Figure 4C). Quantification of inter-fiber distance and fiber volume shown in Figure 4D revealed a significant difference of both parameters in lung nodules following

A

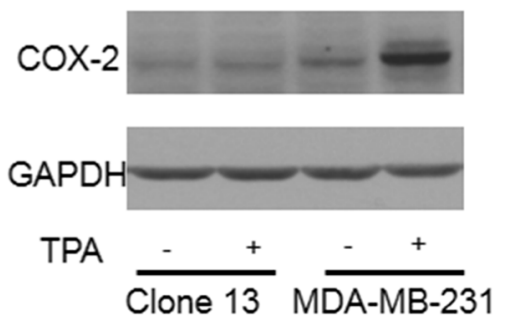

C

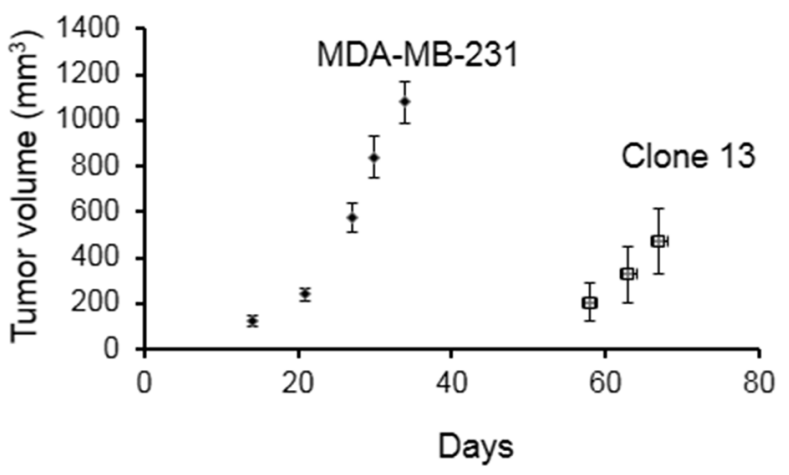

COX-2 reduction. To establish a relationship between COX-2 downregulation in primary tumors and lymph node metastasis, H\&E stained axillary lymph node sections were analyzed for presence of cancer cells. As shown in Figure $4 \mathrm{E}, 80 \%$ of animals were positive for presence of cancer cells in axillary lymph nodes in the MDA-MB-231 tumor group compared to $20 \%$ in the Clone 13 tumor group.

COX-2 downregulation decreased the presence of CAFs in primary tumors. Representative images of $\alpha$-SMA immunostained sections obtained from MDA-MB-231 and Clone 13 tumors are shown in Figures 5A and 5D respectively. Magnified FOVs showing immunostained CAFs and the image segmentation used to identify the fibroblasts are presented in Figures $5 \mathrm{~B}$ and $5 \mathrm{C}$ for the MDA-MB-231 tumor section, and in Figures 5E and 5F for the Clone 13 tumor section. Since smooth muscle cells also express $\alpha$-SMA [28], vessel regions were excluded in the analysis. Quantification of immunostaining identified

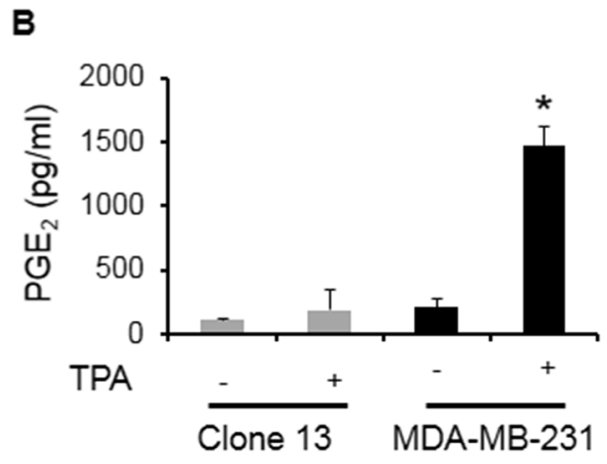

D
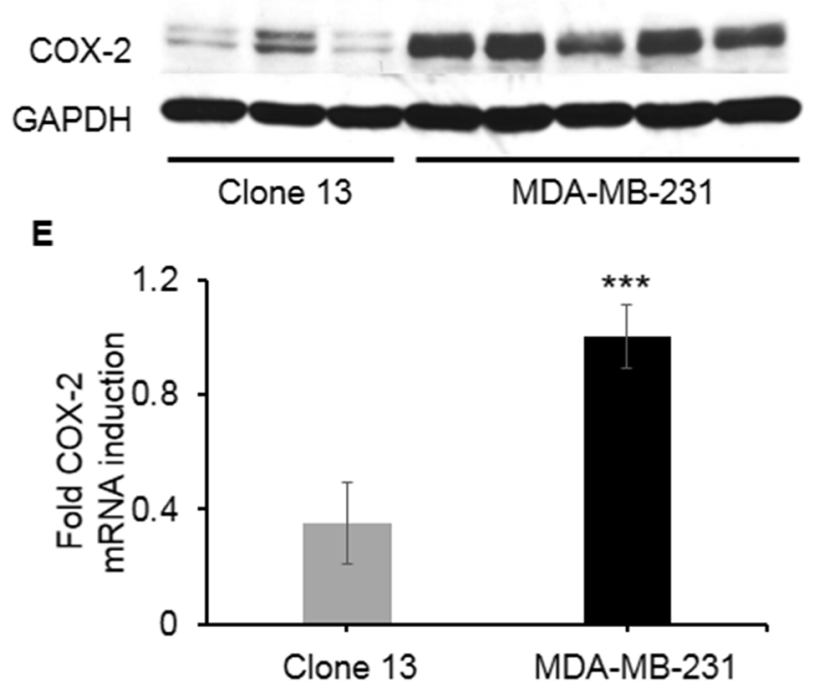

Figure 1: A. COX-2 expression in cells. B. PGE $_{2}$ expression in cells; cells were exposed to $50 \mathrm{nM}$ TPA for $24 \mathrm{~h}$ to induce COX-2 expression. C. Tumor volumes for COX-2 containing parental MDA-MB-231 (N=5) and COX-2 reduced Clone 13 (N=8) tumors; 2 x $10^{6}$ cells were inoculated in $0.1 \mathrm{ml}$ of $8.8 \mathrm{mg} / \mathrm{ml}$ Matrigel. D. Representative immunoblot showing COX-2 expression in MDA-MB-231 and Clone 13 tumors. GAPDH was used as loading control. E. Relative fold change in COX-2 mRNA levels in MDA-MB-231 (N=6) and Clone $13(\mathrm{~N}=4)$ tumors. Values represent Mean $\pm \mathrm{SEM}$. ${ }^{*} \mathrm{p} \leq 0.05 ; * * \mathrm{p} \leq 0.001$ using $\Delta \mathrm{C}_{\mathrm{t}}$ values. 
higher CAFs in MDA-MB-231 tumors compared to Clone 13 tumors, as shown in Figure 5G. Representative $\alpha$-SMA immunoblots obtained from an MDA-MB-231 and a Clone 13 tumor are presented in Figure $5 \mathrm{H}$ and demonstrate the decrease of $\alpha$-SMA expression following COX-2 downregulation.

As shown in representative $5 \mu \mathrm{m}$-thick H\&E and corresponding $\alpha$-SMA immunostained sections obtained from lungs of mice injected with MDA-MB-231 (Figures 6A and 6B) and Clone 13 (Figures 6C and 6D) cells, fewer CAFs were observed in Clone 13 lung nodules that were also typically smaller. Lung nodules obtained from mice injected with MDA-MB-231 or Clone 13 cells in the tail vein revealed a significant correlation between nodule size and the number of CAFs. A significant correlation was observed between the sum of metastatic nodule pixels (reflecting total nodule area) and the sum of strongly positive pixels (reflecting number of CAFs) in lungs obtained from each mouse (Figure 6E), supporting the role of CAFs in the formation of metastasis.

To further establish the role of COX-2 expression in modulating the ECM, we stably overexpressed the coding sequence of COX-2 in SUM-149 breast cancer cells (SUM-149-COX-2FL). Empty vector transduced SUM-149 cells (SUM-149-EV) were used for comparison. Higher basal and TPA-induced COX-2 mRNA and protein expression were confirmed in these cells (Supplementary Figures 1A and 1B). To evaluate
A

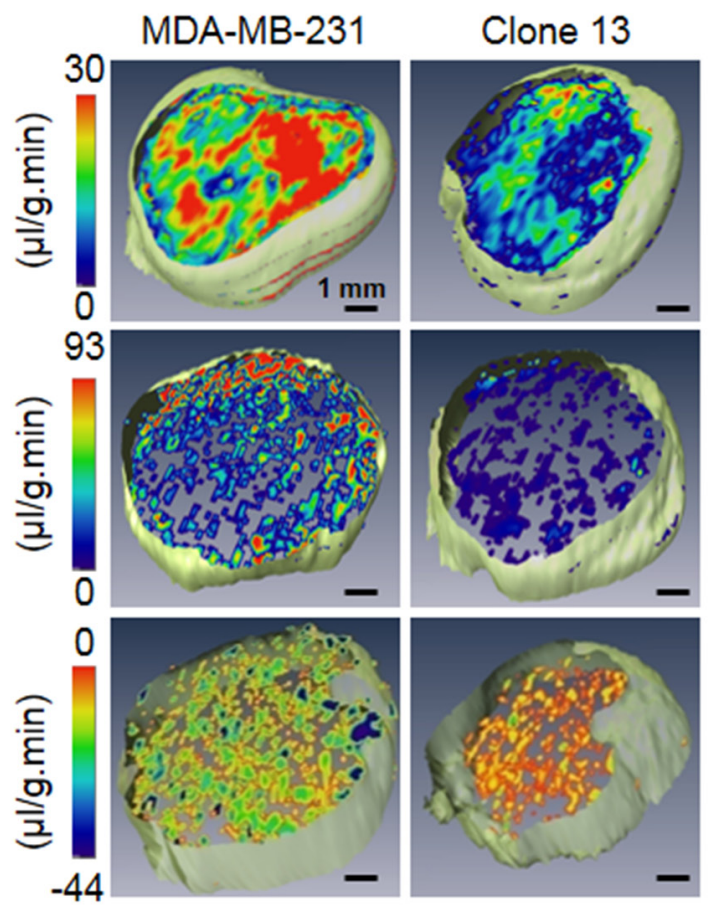

C

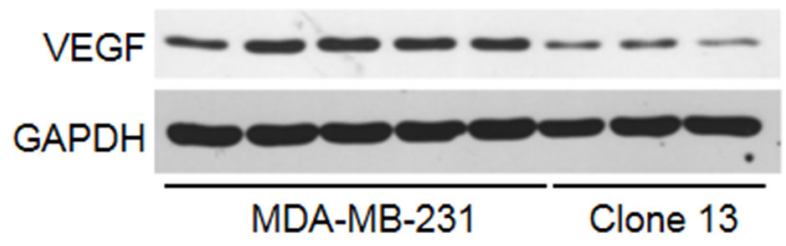

B

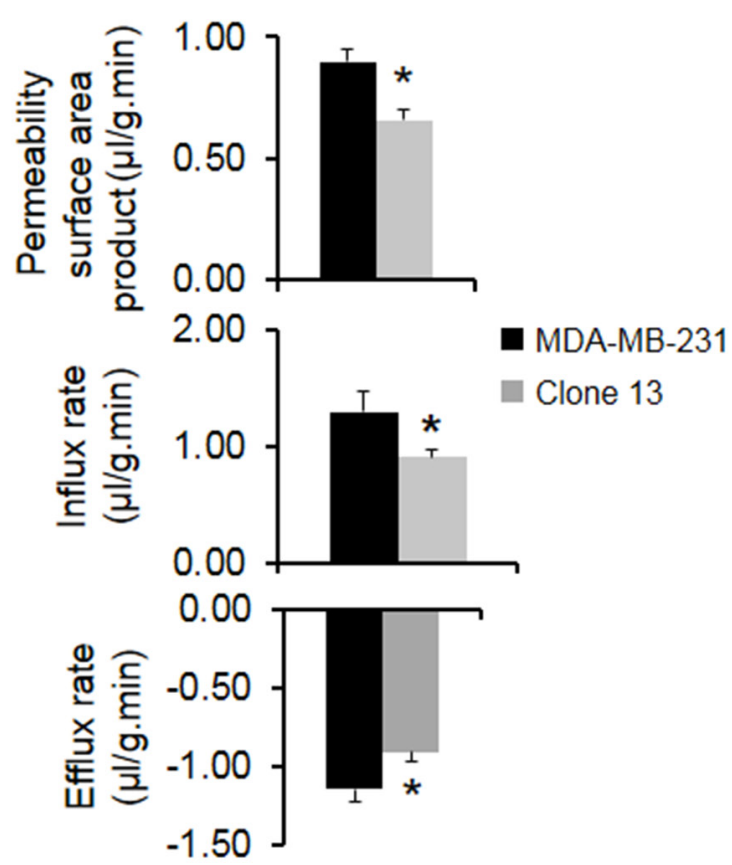

D

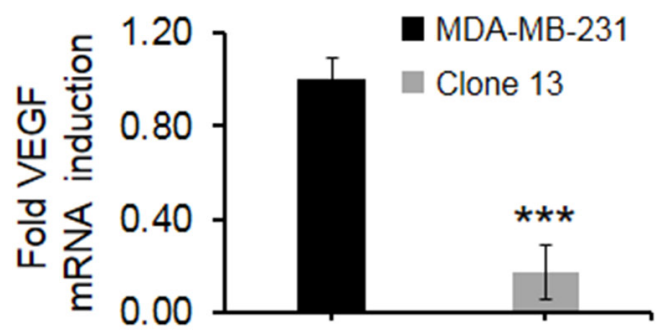

Figure 2: A. Representative 3D maps of permeability surface area product (top), influx rate (middle), and efflux rate (bottom) for high COX-2 expressing parental MDA-MB-231 and COX-2 reduced Clone 13 tumors. B. Quantitative comparisons of permeability surface area product (top), influx rate (middle) and efflux rate (bottom) in high COX-2 expressing parental MDA-MB-231 (N=6) and COX-2 reduced Clone $13(\mathrm{~N}=6)$ tumors. Significantly lower permeability $(\mathrm{p}$-value $=0.003)$, influx rates $(\mathrm{p}$-value $=0.045)$ and efflux rates $(\mathrm{p}$-value $=0.036)$ were observed in COX-2 reduced Clone 13 tumors as compared to COX-2 containing parental MDA-MB-231 tumors. C. Representative immunoblot showing VEGF expression in MDA-MB-231 and Clone 13 tumors. GAPDH was used as a loading control. D. Relative fold change of VEGF mRNA expression in MDA-MB-231 $(\mathrm{N}=6)$ and Clone $13(\mathrm{~N}=4)$ tumors. Values represent Mean $\pm \mathrm{SEM}$. ***p $\leq 0.001$ using $\Delta \mathrm{C}_{\mathrm{t}}$ values 
the functionality of overexpressed COX-2 in the cells we measured secreted $\mathrm{PGE}_{2}$ levels and observed significantly higher basal and TPA-induced $\mathrm{PGE}_{2}$ secretion in these cells (Figure 7A). Tumors derived from these cells also expressed increased mRNA transcript and expressed higher COX-2 protein (Supplementary Figures $1 \mathrm{C}$ and $1 \mathrm{D})$.

To further validate the role of COX-2 expression in modifying the ECM, SHG microscopy was performed on sections obtained from SUM-149-EV and SUM149-COX-2FL cell derived tumors. Denser Coll fibers were observed in SUM-149-COX-2FL tumor sections compared to SUM-149-EV tumor sections as shown in the representative Coll fiber images in Figure 7B. Quantification of fiber volume and inter-fiber distance confirmed a significant increase of fiber volume and a significant decrease of inter-fiber distance with COX-2 overexpression (Figure 7C).

COX-2 overexpression in SUM-149 tumors increased the number of CAFs detected in the tumors as shown in the representative images obtained at $1 \mathrm{X}$ and 20X from SUM-149-EV and SUM-149-COX-2FL tumor sections stained for $\alpha$-SMA (Figure 7D). This increase was confirmed following quantification of immunostained sections (Figure 7E). To further understand the role of COX-2 on changes in Coll fiber content, human mammary fibroblasts (HMFs) were exposed to $\mathrm{PGE}_{2}$ and Col1A1 expression levels were determined. Exposure to $\mathrm{PGE}_{2}$ resulted in increased Col1A1 expression (Supplementary Figure 2).
A

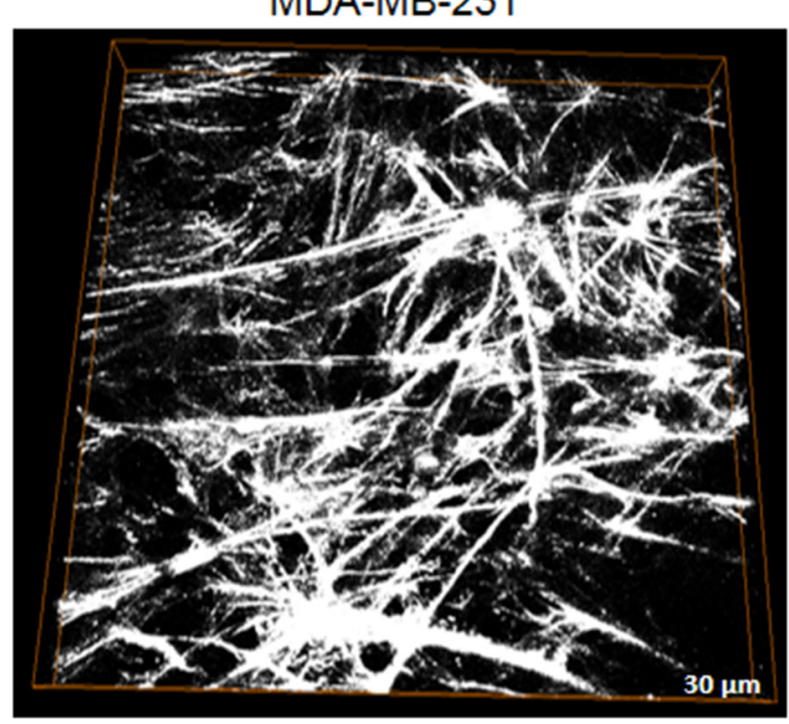

Clone 13

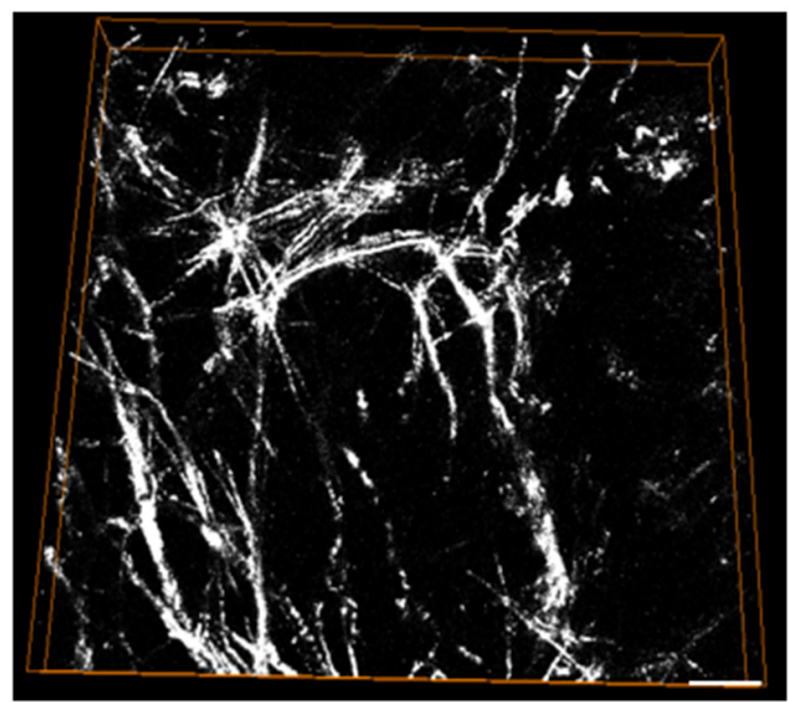

B

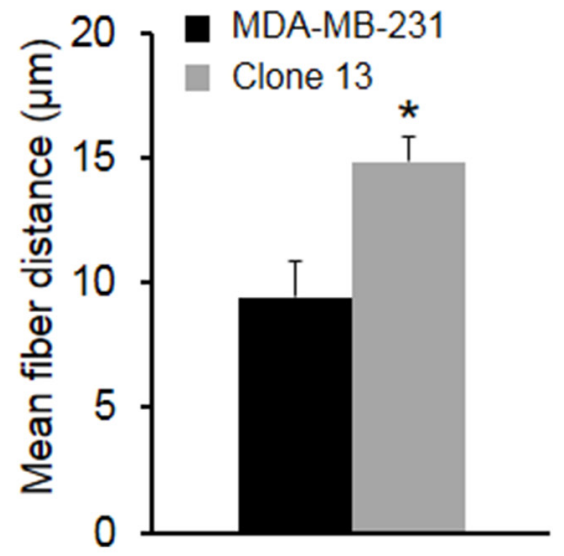

MDA-MB-231

Clone 13

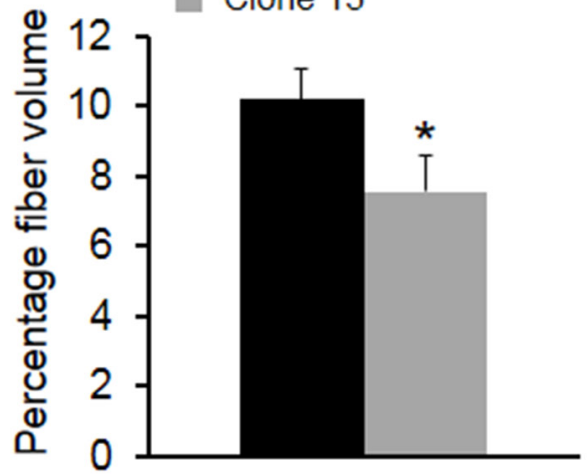

Figure 3: A. 3D visualization of Coll fibers in COX-2 containing parental MDA-MB-231 and COX-2 reduced Clone 13 tumors. The FOV image size was $334.91 \times 334.91 \times 15 \mu \mathrm{m}^{3}$ with a voxel size of $0.66 \times 0.66 \times 1 \mu \mathrm{m}^{3}$. B. Quantification of Coll fiber volume and fiber distribution. COX-2 reduced Clone 13 tumors $(\mathrm{N}=7)$ had significantly larger inter-fiber distance and significantly lower percent fiber volume compared to COX-2 containing parental MDA-MB-231 tumors (N=5). Values represent Mean \pm SEM. *p $\leq 0.05$. 
COX-2 overexpression in SUM-149 cells did not significantly increase spontaneous metastasis in the axillary lymph nodes (data not shown). Both SUM149-EV and SUM-149-COX-2FL cells formed emboli in the vasculature, and pulmonary metastasis. COX-2 overexpression did not increase the size of the emboli or pulmonary metastasis, following intravenous injection of the cells (Figures 7F and 7G). COX-2 overexpression did, however, significantly increase $(p<0.05)$ the number of pulmonary metastatic nodules. Mean \pm SEM values of the number of pulmonary metastatic nodules established by SUM-149-COX-2FL cells was $5.4 \pm 1.56, \mathrm{n}=5$, versus $2 \pm 0.83, \mathrm{n}=5$, established by SUM-149-EV cells.

\section{DISCUSSION}

Here we have shown that downregulating COX2 expression significantly impacted ECM structure, by reducing Coll fiber volume, and ECM function, by altering permeability and macromolecular transport, in MDA-MB-231 tumors. Decrease of VEGF was identified as a mechanism by which vascular permeability decreased. COX-2 downregulation reduced the ability of these TNBC cells to form metastatic lung nodules and to metastasize to lymph nodes. Additionally, a significant decrease of Coll fibers was observed in metastatic lung nodules established by COX-2 downregulated cells. To identify the cause of
A

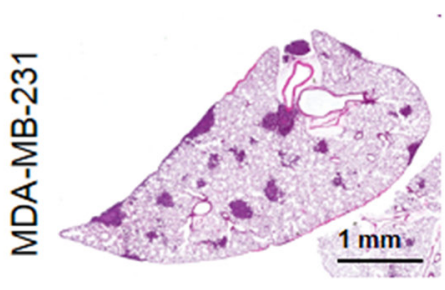

C.

MDA-MB-231
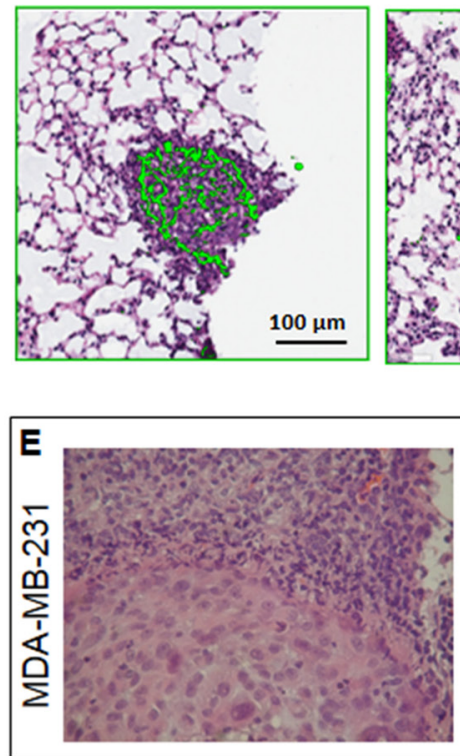

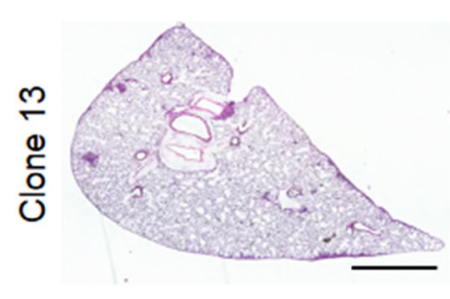

D

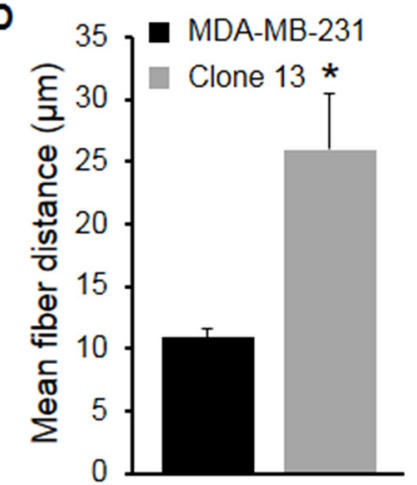

B
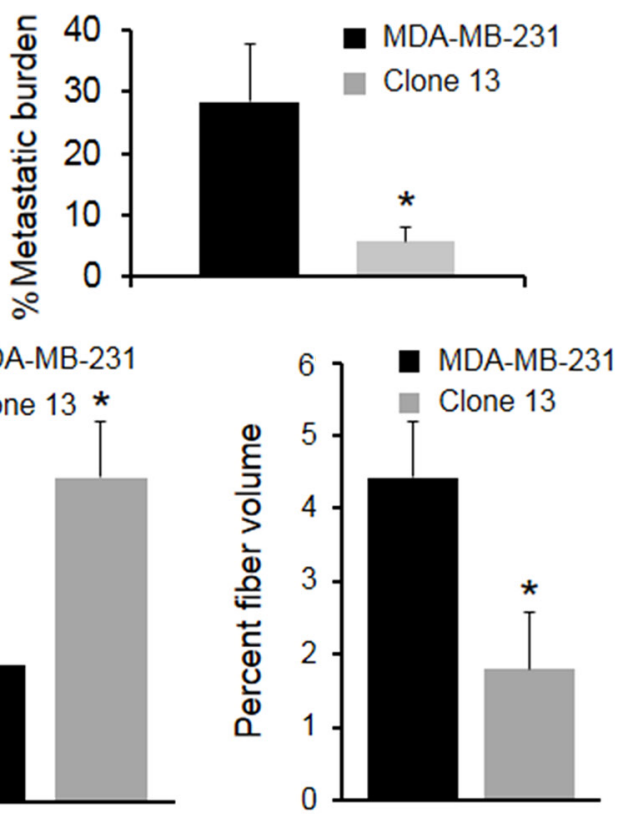

Figure 4: A. Representative examples of H\&E stained tumor sections of lungs obtained from mice intravenously injected with $10^{6} \mathrm{MDA}-$ MB-231 or Clone 13 cells. B. Metastatic burden was calculated as [(Total area of metastatic foci in $\left.\mu \mathrm{m}^{2}\right) /\left(\mathrm{Total}\right.$ lung area in $\left.\left.\mu \mathrm{m}^{2}\right)\right] \mathrm{x} 100$. Metastatic burden from MDA-MB-231 injected mice $(\mathrm{N}=5)$ was significantly higher $(\mathrm{p}=0.059)$ compared to metastatic burden from Clone 13 injected mice $(\mathrm{N}=3)$. Values represent Mean $\pm \mathrm{SEM} .{ }^{*} \mathrm{p} \leq 0.06$. C. Representative images of Coll fiber distribution in metastatic lung nodules obtained with SHG microscopy overlaid on the corresponding H\&E stained region, from mice intravenously injected with $10^{6}$ MDA-MB-231 or Clone 13 cells. D. Quantification of Coll fiber volume and fiber distribution in lung nodules. Lung nodules obtained from mice injected with COX-2 reduced Clone $13(\mathrm{~N}=3)$ cells had significantly larger inter-fiber distance $(\mathrm{p}$-value $=0.053)$ and significantly lower percent fiber volume $(\mathrm{p}$-value $=0.049)$ compared to COX-2 containing parental MDA-MB-231 mice $(\mathrm{N}=5)$. Values represent Mean \pm SEM. ${ }^{*} p \leq 0.05$. E. Representative photomicrographs of H\&E stained sections of lymph nodes with cancer cells. Four of five MDAMB-231 tumor-bearing mice had cancer cells detected in the axillary lymph nodes and one of four Clone 13 tumor-bearing mice had cancer cells detected in the axillary lymph nodes. 
Coll fiber reduction, we determined the number of CAFs in primary tumors and metastatic lung nodules. Consistent with the decrease of Coll fibers, a significant reduction of CAFs was observed in COX-2 downregulated MDAMB-231 tumors with a similar trend in metastatic nodules. A significant correlation was observed between the size of the nodule and the number of CAFs, identifying the importance of activated fibroblasts in the formation of metastasis, and the role of COX-2 in activating fibroblasts.

Downregulating COX-2 resulted in a significant delay of tumor onset of several days as well as slower growth. Although significant, the delay was not as profound as the delay of several weeks observed when COX-2 was completely silenced [17] further confirming the critical dependence of tumors on the prostaglandins produced by the enzyme for growth and progression [3].

Prostanoids produced by COX-2 such as $\mathrm{PGE}_{2}$ have been observed to mediate changes in angiogenesis [29-31], and anti-inflammatory agents have been found to have an antiangiogenic effect [31, 32]. Silencing of COX-2 in MDA-MB-231 cells downregulated several angiogenesis related transcripts [19]. COX2-silenced MDA-MB-231 cells failed to promote the characteristic self-association patterns of endothelial cells in a co-culture model [19]. Here, a significant decrease of permeability and VEGF was observed in COX-2 downregulated MDA-MB-231 tumors. PGE 2 is a major regulator of vascular permeability $[31,33]$, and
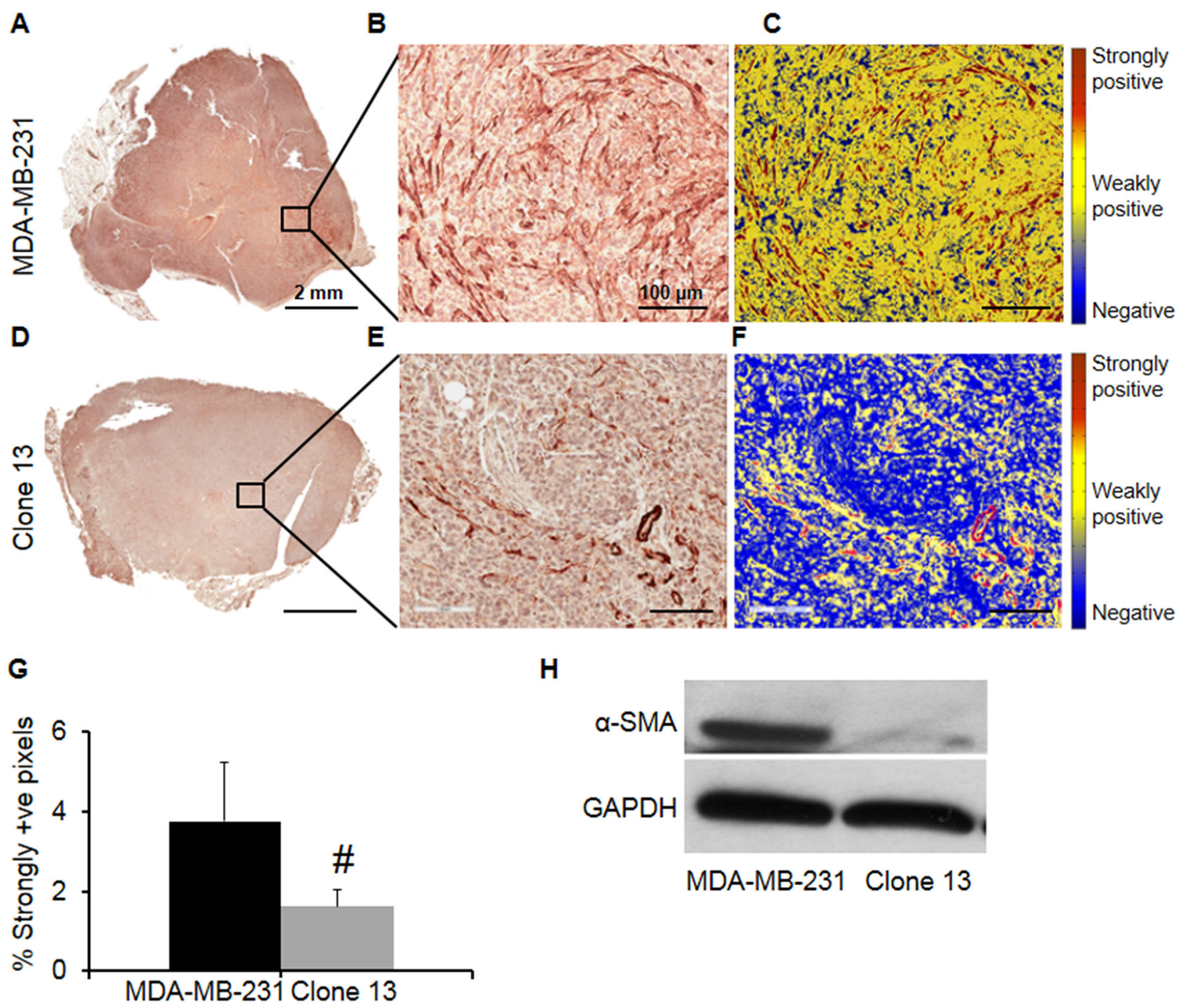

H

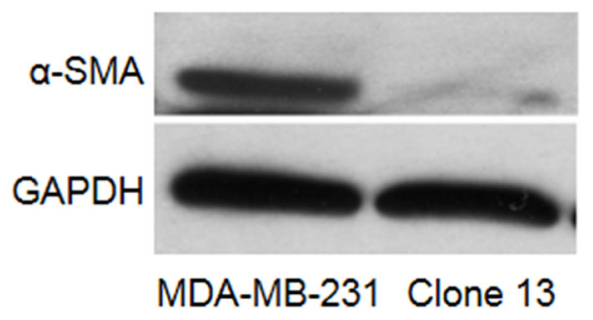

Figure 5: Representative images of $\alpha$-SMA immunostained sections obtained from A. MDA-MB-231 and D. Clone 13 tumors. Magnified FOVs showing immunostained CAFs and the accuracy of the algorithm in identifying CAFs are presented in B. and C. for the MDA-MB-231 tumor section and in $\mathbf{E}$. and $\mathbf{F}$. for the Clone 13 tumor section. G. Quantification of immunostaining identified the presence of higher CAFs in MDA-MB-231 tumors $(\mathrm{N}=5)$ compared to Clone 13 tumors $(\mathrm{N}=6)$. Values represent Mean $\pm \mathrm{SEM}$. \#p $\leq 0.084$. H. Representative $\alpha$-SMA immunoblot obtained from an MDA-MB-231 and a Clone 13 tumor. GAPDH was used as a loading control. 
the decrease of vascular permeability is consistent with the decrease of $\mathrm{PGE}_{2}$ and VEGF production observed in these tumors. Interestingly, macromolecular transport through the ECM was also significantly reduced with COX-2 downregulation, indicating functional modulation of the ECM by COX-2.

Previous studies have shown a marked alteration of the degradome and invasion associated transcripts,

A

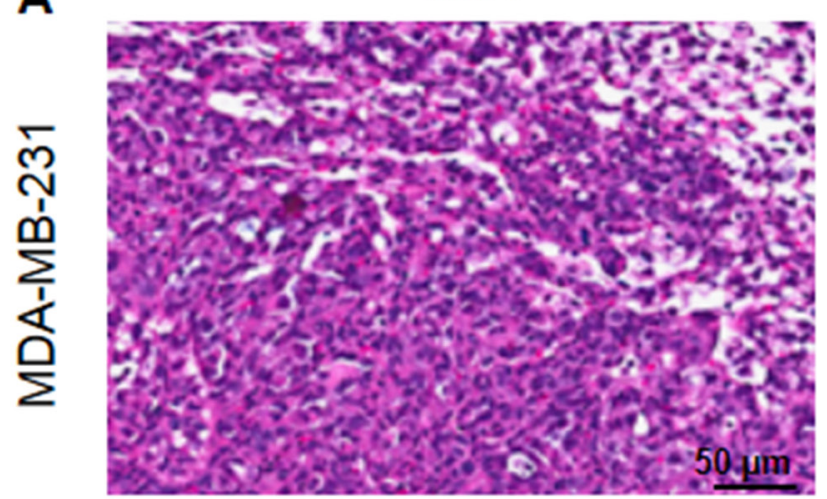

C

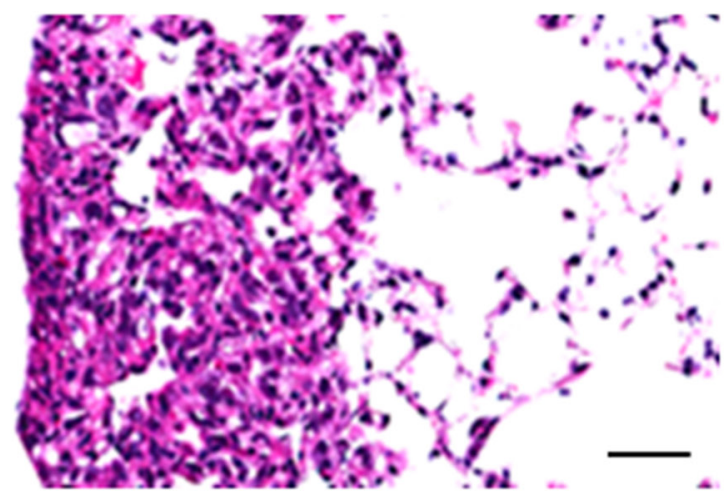

E including a several fold down regulation of MMP1 [19], following COX-2 silencing in MDA-MB-231 cells. Here, COX-2 downregulated MDA-MB-231 cells showed a marked decrease in the ability to invade and colonize the lungs. Colonization of lymph nodes was also attenuated. These data further support the use of COX-2 inhibition to attenuate the metastatic cascade for those tumors that are COX-2 dependent.
B

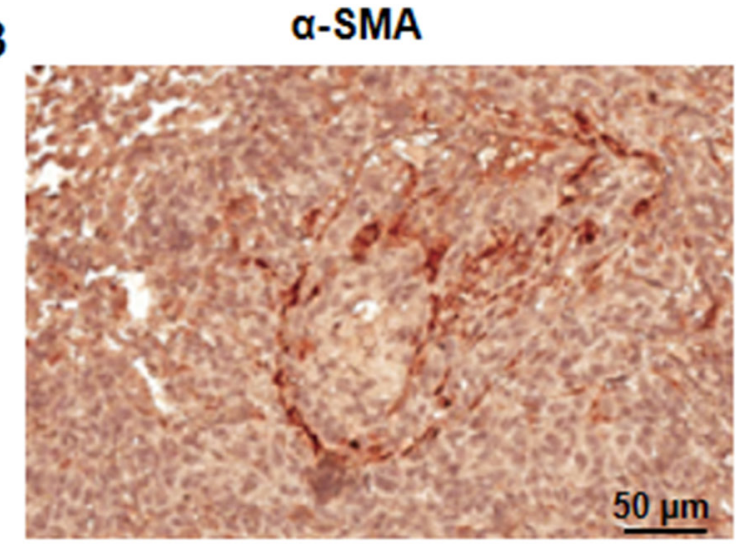

D

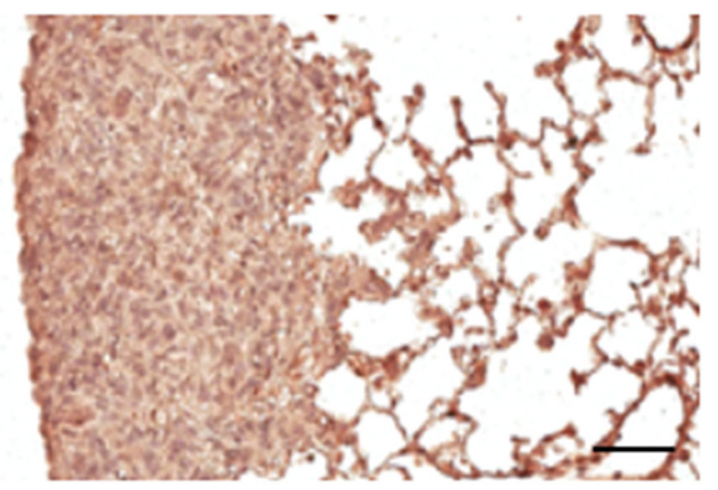

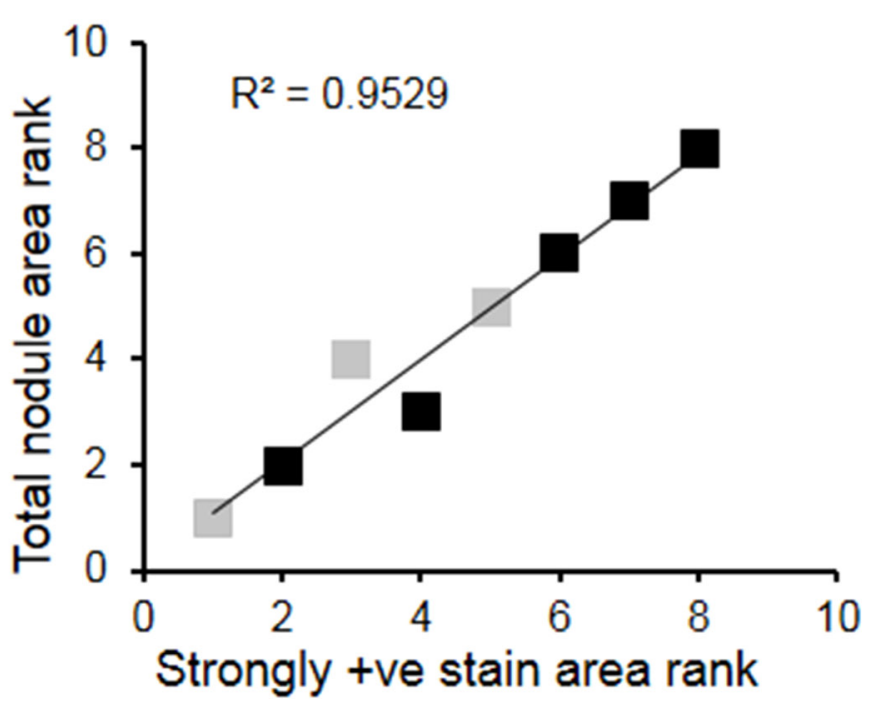

MDA-MB-231

Clone 13

Figure 6: Representative $5 \mu \mathrm{m}$-thick H\&E and corresponding $\alpha$-SMA immunostained sections obtained from lungs of mice injected with A, B. MDA-MB-231 (N=5) and C, D. Clone $13(\mathrm{~N}=3)$ cells. E. Spearman correlation between sum of metastatic nodule pixels (reflecting total nodule area) and sum of strongly positive pixels (reflecting number of CAFs) in lungs obtained from each mouse. A significant correlation was observed supporting the role of CAFs in the formation of metastasis. 
We observed that the COX-2 downregulated MDAMB-231 tumors had significantly sparser Col1 fiber distribution. These data further confirm earlier observations that COX-2 pharmacological inhibition reduces collagen deposition during mammary gland involution [22]. Col1 fiber density and orientation are increasingly being linked to breast cancer metastasis [25] and increased collagen content may contribute to the negative prognostic value of COX-2 expression in breast cancer patients [34, 35]. Col1 fibers were identified as channels that facilitate the ameboid movement of MDA-MB-231 cancer cells [36]. Reduced Coll fibers have been previously associated with decreased macromolecular transport [37], and the reduced Coll fibers in COX-2 downregulated tumors may have altered macromolecular transport in these tumors. Reduction of Coll fibers was observed in metastatic nodules established by Clone 13 cancer cells following intravenous injection, suggesting that Coll fibers are also important in the establishment of metastasis following extravasation.

Further confirmation of the role of COX-2 in modifying the ECM was evident from the significantly increased Coll fiber density and volume observed in tumors derived from COX-2 overexpressing SUM-149 cells. COX2 overexpression resulted in a significant increase of the
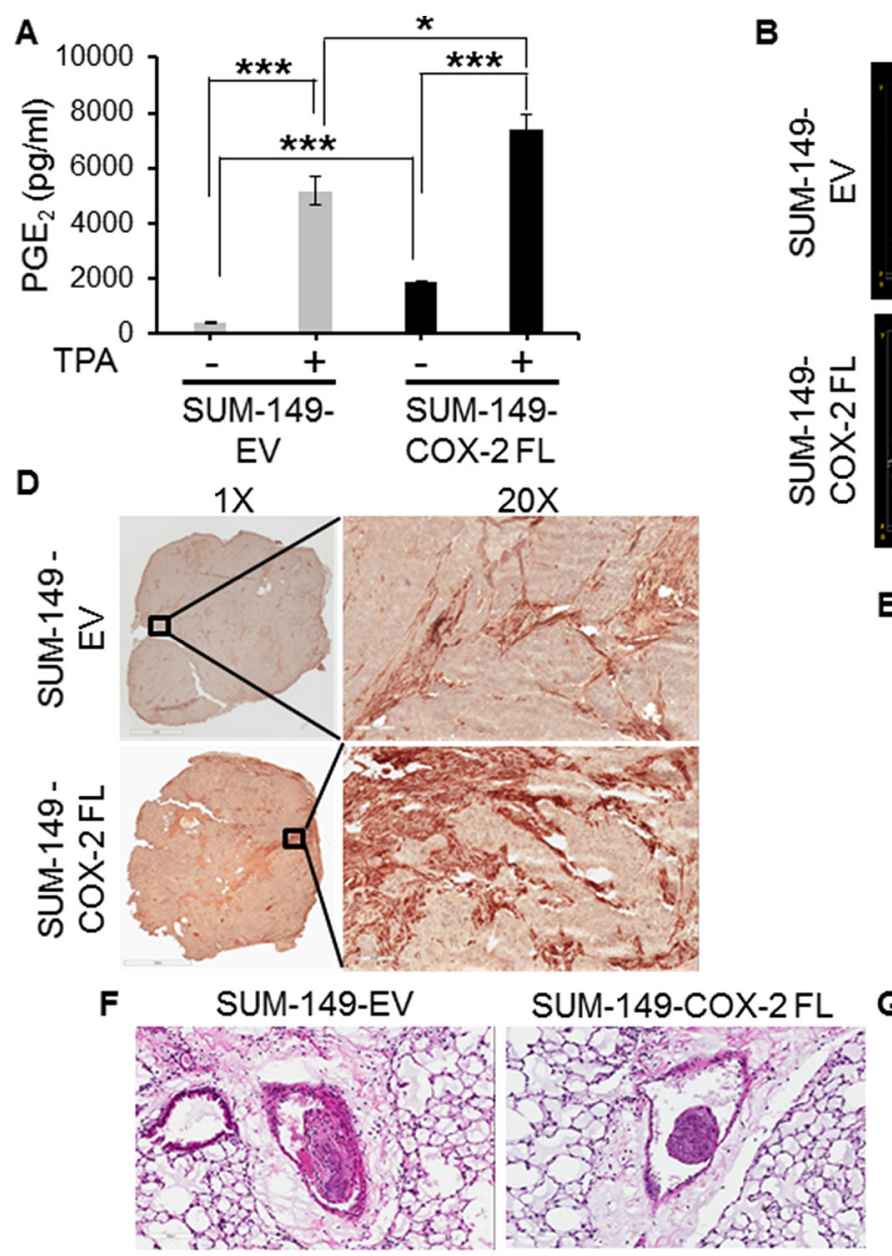

B

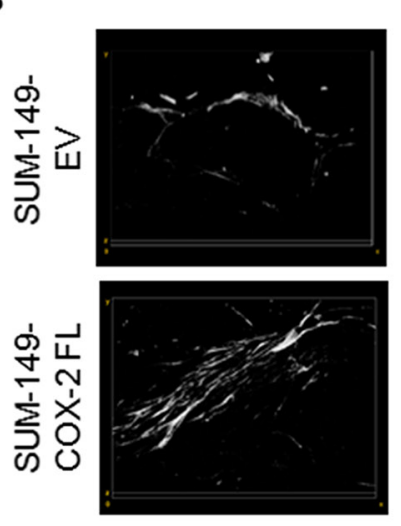

E
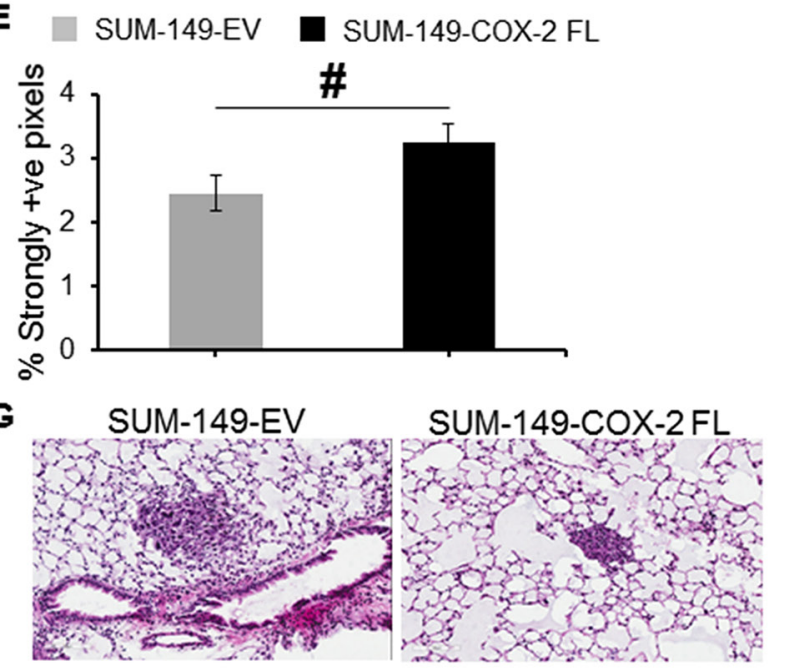

Figure 7: A. PGE 2 expression in COX-2 overexpressing cells; cells were exposed to 50nM TPA for 24hrs to induce COX-2 expression. Values represents Mean \pm SEM from four independent experiments; $* p \leq 0.005$. B. 3D visualization of Coll fibers in empty vector expressing and COX-2 overexpressing SUM-149 tumors. The FOV image size was $423.5 \times 423.5 \times 12 \mu m^{3}$. C. Quantification of Coll fiber volume and fiber density. COX-2 overexpressing tumors $(\mathrm{N}=5)$ had significantly lower inter-fiber distance and significantly higher percent fiber volume compared to SUM-149-EV tumors $(\mathrm{N}=5)$. Values represent Mean \pm SEM. $* \mathrm{p} \leq 0.05$, $* * \mathrm{p} \leq 0.005$. D. Representative images of $\alpha$-SMA immunostained sections obtained from SUM-149-EV (top) and SUM-149-COX-2FL (bottom) tumors. Magnified FOVs at 20X show immunostained CAFs for SUM-149-EV (top) and SUM-149-COX-2FL (bottom) tumors. E. Quantification of immunostained sections identified a higher number of CAFs in SUM-149-COX-2FL tumors ( $\mathrm{N}=5)$ compared to SUM-149-EV tumors (N=5). Values represent Mean \pm SEM. \# p=0.06. F. Representative high resolution 20X H\&E images of lung section showing emboli formation following intravenous injection of SUM-149-EV cells (left) and SUM-149-COX-2FL cells (right). G. Representative high-resolution 20X images of lung showing pulmonary metastatic foci following intravenous injection of SUM-149-EV cells (left) and SUM-149-COX-2FL (right). 
number of pulmonary metastases, further supporting the role of COX-2 in establishing metastasis.

We used the expression of $\alpha$-SMA to detect CAFs [38] in primary tumors and metastatic lung nodules. CAFs are a major source of Coll fibers in the tumor stroma and contribute to the reactive desmoplastic tumor stroma [27]. CAFs play an active role in breast cancer metastasis $[39,40]$. Here, for the first time, we observed that COX2 downregulation in TNBC cells resulted in a significant decrease of CAFs in primary tumors derived from these cells, and in metastatic lung nodules. Conversely, COX2 overexpression resulted in an increase of CAFs in primary SUM-149 tumors derived from these cells. The COX-2 dependent increase or decrease of CAF numbers may primarily explain the decrease in Coll fiber content with COX-2 downregulation and the increase in Coll fiber content with COX-2 overexpression. These data are consistent with a significant reduction of Coll fibers observed following treatment of tumors with the antifibrotic agent Pirfenidone that eliminated CAFs [41]. $\mathrm{PGE}_{2}$ formed by COX-2 also increased Col1A1 expression in HMFs [42], although opposing effects have also been observed depending upon the type of fibroblast investigated [43].

Our studies were performed with TNBC cells, but future studies with ER/PR/HER2 positive breast cancer cells should further expand our understanding of the role of COX-2 in modifying the ECM and CAF numbers.

The role of CAFs in the establishment of metastasis was evident from the strong correlation between the size of the nodule and the number of CAFs present in the nodule. These results are also consistent with recent studies identifying the symbiosis between cancer cells and CAFs in tumor progression [44]. In addition to identifying the role of COX-2 in activating fibroblasts, our data suggest that including CAF immunostaining of breast cancer specimens may assist in identifying more aggressive cancers. The data also support disrupting cancer cell-fibroblast interactions as a strategy to arrest tumor growth and metastatic dissemination.

Collectively these data expand our insights into the role of COX-2 in breast cancer and its impact on the structure and function of the ECM. These insights are important as changes in the ECM and CAFs may occur during the course of treatments that upregulate COX-2 [45]. Our data identify a close dependence between COX2 expression and the number of CAFs in primary tumors and metastatic nodules, and identify cancer-cell fibroblast signaling disruption as a potential treatment strategy to prevent metastatic dissemination.

\section{MATERIALS AND METHODS}

\section{Stable expression of the COX-2 shRNA- containing plasmid in MDA-MB-231 cells}

MDA-MB-231 breast cancer cells were obtained from ATCC (ATCC, Manassas, VA) and maintained in RPMI 1640 medium (Mediatech, Manassas, VA) supplemented with $10 \%$ fetal bovine serum (SigmaAldrich, St. Louis, MO). The COX-2 shRNA-coding plasmid was constructed and placed under the control of the U6 promoter as previously described [17]. Individual clones were selected for G418 resistance and analyzed for $\mathrm{PGE}_{2}$ production from the supernatant using the $\mathrm{PGE}_{2}$ enzyme immunoassay (EIA) Kit-Monoclonal as described by the manufacturer (Cayman Chemical, Ann Arbor, MI). Cells were induced for COX-2 expression by exposure to $50 \mathrm{nM}$ TPA for twenty-four hours. Clone 13 cells were selected based on their significantly reduced basal and inducible COX-2 expression and $\mathrm{PGE}_{2}$ production.

\section{Overexpression of COX-2 in SUM-149 breast cancer cells}

SUM-149 breast cancer cells were obtained from Asterand (Asterand, Inc., Detroit, MI) and maintained in Ham's F12 medium (SIGMA, St. Louis, MO) with 5\% calf serum, insulin $(5 \mu \mathrm{g} / \mathrm{ml})$, and hydrocortisone $(1 \mu \mathrm{g} /$ $\mathrm{ml})$. An $\sim 1.8 \mathrm{~Kb}$ region of the coding sequence of the human COX-2 gene (NM_000963.3) was PCR amplified and cloned into the PCR2.1 Topo vector (Invitrogen, Waltham, MA) and later subcloned between Xho1 and Kpn1 restriction sites in the multiple cloning site (MCS) of a pHAGE-pGK-MCS-Gtx-GFP lentivirus vector. 293T cells (ATCC, Manassas, VA) were co-transfected with the pHAGE-COX-2FL-Gtx-GFP plasmid, the $\triangle \mathrm{R} 8.2-$ packaging plasmid, and a plasmid expressing vesicular stomatitis virus glycoprotein (VSVG) to produce virions. Supernatant containing virions was added to SUM-149 breast cancer cells to derive cells stably expressing the COX-2 gene (SUM-149-COX-2FL). An empty vector without the gene was used to derive control cells (SUM149-EV). Stable increase of COX-2 expression was verified by PCR and western blot analysis.

\section{Effect of $\mathrm{PGE}_{2}$ on Col1A1 expression in HMFs}

HMFs kindly provided by Dr. Gary Luker, University of Michigan, Ann Arbor, were cultured in DMEM medium containing $10 \%$ fetal bovine serum (SIGMA, St. Louis, MO). For Col1A1 protein expression, HMFs were seeded in three $100 \mathrm{~mm}$ dishes at $1.3 \times 10^{6}$ cells per dish. Once the cells attached to the dish, cells were serum starved for twenty-four hours. At the end of serum starvation, $\mathrm{PGE}_{2}$ was added to two dishes at $3 \mathrm{ng} / \mathrm{ml}$ or 30 $\mathrm{ng} / \mathrm{ml}$ in serum-free DMEM for an additional forty-eight hours. Untreated and $\mathrm{PGE}_{2}$ treated cells were analyzed for Col1A1 expression.

\section{Protein and mRNA expression}

Expression levels of COX-2, $\alpha$-SMA, Col1A1, and VEGF were determined by immunoblotting after blocking with 5\% nonfat milk, with goat anti-COX-2 antibody 
(1:500, Cayman Chemical, Ann Arbor, Michigan), a monoclonal antibody against $\alpha$-SMA (Clone 1A4, 1:1000), a rabbit polyclonal antibody against Col1 A1 (ORIGENE, Rockville, MD), or an anti-VEGF polyclonal antibody (1:2000, Millipore Temecula, CA), and visualized with HRP (horseradish peroxidase)-conjugated secondary antibodies using the SuperSignal West Pico Chemiluminescent substrate kit (Thermo Scientific, Rockford, IL). Monoclonal anti-GAPDH antibody (1:50,000 dilution, Sigma-Aldrich) was used as loading control.

Total RNA was isolated from cells and tumor samples using the QIAshredder and RNeasy Mini kit (Qiagen, Valencia, CA). cDNA was prepared using the iScript cDNA synthesis kit (Bio-Rad). cDNA samples were diluted 1:10 and real-time PCR was performed using IQ SYBR Green supermix and gene specific primers in the iCycler real-time PCR detection system (Quanta Bioscience, Gaithersburg, MD). All primers were designed using Beacon designer software 7.8 (PREMIER Biosoft, Palo Alto, CA). The expression of target RNA relative to the housekeeping gene HPRT1 was calculated based on the threshold cycle $(\mathrm{Ct})$ as $\mathrm{R}=2^{-\Delta(\Delta \mathrm{Ct})}$, where $\Delta \mathrm{C}_{\mathrm{t}}=\mathrm{C}_{\mathrm{t}}$ of target $-\mathrm{C}_{\mathrm{t}}$ of HPRT1.

\section{Tumor studies}

Tumors derived from parent MDA-MB-231 and Clone 13 cells, with lower basal and inducible COX2 expression levels and SUM-149 cells expressing an empty vector (SUM-149-EV) or overexpressing COX-2 (SUM-149-COX-2FL) were studied in vivo. Approximately $2-3 \times 10^{6}$ cancer cells in $0.05 \mathrm{ml}$ of Hanks balanced salt solution (HBSS) (Sigma-Aldrich, St. Louis, MO) were inoculated in the mammary fat pad of female severe combined immunodeficient (SCID) mice. Growth curves were obtained using cells inoculated in $0.05 \mathrm{ml}$ of Matrigel solution $(8.8 \mathrm{mg} / \mathrm{ml}$ ) (SigmaAldrich). Orthotopic tumors were used to investigate the relationship between COX-2 expression, macromolecular transport using MRI and Coll fiber density and volume using SHG microscopy. Lymph nodes excised from euthanized tumor-bearing mice were fixed in formalin, embedded in paraffin, sectioned at $5 \mu \mathrm{m}$ thickness, and stained with H\&E to evaluate for spontaneous metastasis. Separate sets of mice were injected intravenously with $10^{6}$ MDA-MB-231 or Clone 13 cells in $0.05 \mathrm{ml}$ of HBSS. Tail vein injected mice were euthanized eight weeks later and metastatic burden and Coll fibers in the metastatic nodules were evaluated from $0.5 \%$ agarose infused lungs that were fixed in formalin, embedded in paraffin, and sectioned.

All surgical procedures and animal handling were in accordance with protocols approved by the Johns Hopkins University Institutional Animal Care and Use Committee.

\section{MRI}

Mice were imaged once tumor volumes were approximately $400-500 \mathrm{~mm}^{3}$. Mice were anesthetized, and a home-built catheter was inserted in the tail vein to inject the macromolecular contrast agent, albumin-gadoliniumdiethylenetriaminepentaacetic acid (albumin-GdDTPA). MRI was performed on a $4.7 \mathrm{~T}$ Bruker spectrometer using a home built solenoid coil placed around the tumor. The respiration rate was monitored, and an isoflurane mask was used to maintain stable anesthesia during the $140 \mathrm{~min}$ of MRI scan time. The MRI acquisition was performed on volume-matched tumors as previously described [46]. Briefly, multi-slice relaxation rates $\left(\mathrm{T}_{1}{ }^{-1}\right)$ were acquired using a saturation recovery technique with fast- $\mathrm{T}_{1}$ SNAPSHOT FLASH imaging (flip angle = 10 degrees, echo time $=2 \mathrm{~ms}$ ). At the end of the MRI acquisition, blood $\mathrm{T}_{1}$ was determined from 20 microliters drawn from the tail vein. Images of the central 4 slices (slice thickness of $1 \mathrm{~mm}$ ) of the tumor were acquired (128 $\mathrm{x} 128$ matrix, $16 \mathrm{~mm}$ field of view, number of average $=$ 8) for three relaxation delays (100, 500 and $1000 \mathrm{~ms})$. A multislice map of completely relaxed magnetization $\left(\mathrm{M}_{0}\right.$ map) was also acquired with a recovery time of seven seconds. The in-plane resolution of the MR images was $125 \mu \mathrm{m}$ x $125 \mu \mathrm{m}$. Macromolecular transport parameters were measured from quantitative $T_{1}$ maps obtained before and following intravenous administration of albuminGdDTPA (500 mg/kg dose). Images were acquired in two "phases". The "early phase" acquisition images included a pre-contrast image, and a 3-minute post-contrast image that was repeated every 5 minutes over the initial 30 minutes to characterize the tumor vascular volume and permeability surface area product. A second block of MR data, acquired up to 140 minutes post-contrast, was used to characterize the macromolecular transport parameters through the ECM. These transport parameters included the number of draining and pooling voxels, draining and pooling rates, and exudate volumes, derived as previously described [46]. A draining voxel was defined as a voxel in which the contrast agent accumulated at a rate lower than the permeability surface area product (PS), and a pooling voxel was one in which the contrast agent accumulated at a rate higher than the PS. After identifying the draining and pooling voxels, the influx and efflux rates were calculated. All quantification analysis was done in a home-built program written in IDL (ITT Exelis Visual Information Solutions, Herndon, VA) and AFNI (NIH software).

\section{Microscopy}

Multiphoton microscopy was used to detect the SHG signal from Coll fibers in $1 \mathrm{~mm}$ thick fresh tissue slices. SHG imaging was performed as previously described [47]. Briefly, we used a $25 \times / 0.8$ LD LCI PlanApo multi-immersion lens on a Zeiss 710 LSM NLO 
Meta multiphoton microscope (Carl Zeiss MicroImaging, Inc, Thornwood, NY). 3-dimensional (3D) image stacks were acquired from at least 10 randomly selected fields of view (FOVs) for each tumor. Following optical imaging, 5 $\mu \mathrm{m}$ thick adjacent sections were obtained from the optical slice and stained with H\&E or with anti- $\alpha$-SMA antibody as detailed in the immunostaining section.

Inflated lungs from mice in the experimental metastasis study were fixed with formalin. Five $\mu \mathrm{m}$ thick sections were obtained and stained with H\&E or with anti- $\alpha$-SMA antibody. Multiphoton microscopy was used to detect SHG signal from Coll fibers in the H\&E sections of the lungs from at least five randomly selected FOVs from each lung, using an Olympus Laser Scanning FV1000MPE multiphoton microscopy (Olympus Corp., Center Valley, PA) with a 25Xw/1.05XLPLN MP lens.

Coll fiber distribution analysis was performed as previously described [47] by quantifying inter-fiber distance and percent fiber volume using a customized program written in Matlab (MATLAB 7.4.0, The MathWorks, Natick, MA).

Metastatic burden was calculated as [(Total area of metastatic foci in $\left.\mu \mathrm{m}^{2}\right) /\left(\right.$ Total lung area in $\left.\left.\mu \mathrm{m}^{2}\right)\right] \mathrm{x} 100$ from high-resolution digital scans of the H\&E sections obtained using ScanScope (Aperio, Vista, CA). Images were processed with ImageScope software (Aperio). Coll fiber distribution in these sections was performed as described earlier using SHG microscopy data acquired from sizematched metastatic lung nodules to avoid nodule size bias.

\section{Fibroblast immunostaining}

Immunohistochemistry of tumor sections was performed using the streptavidin-peroxidase technique and the DAKO EnVision System (Dako Cytomation, Hamburg, Germany) as previously described [48] using the alkaline conjugated monoclonal anti- $\alpha$-SMA antibody (clone 1A4) primary antibody (Sigma; 1:200 dilution, $4^{\circ} \mathrm{C}$ overnight).

High-resolution digital scans of the immunostained sections were obtained using ScanScope (Aperio). Images were processed and nuclei and membrane intensity quantified with ImageScope software using the algorithm supplied by the manufacturer (Aperio). The number of CAFs was quantified by computing the fraction of intensely stained pixels in the FOVs. Intensities from vessel regions were excluded for quantification.

\section{Statistical analysis}

Statistical significance was determined using an unpaired Students t-test performed using Microsoft Office Excel 2010 (Microsoft, Redmond, WA). P values $\leq 0.05$ were considered significant unless otherwise stated. To determine if the total metastatic nodule area was associated with strong $\alpha$-SMA expression, i.e. the number of activated fibroblasts, we computed the Spearman rank correlation coefficient between these data for both tumor types.

\section{Abbreviations}

Albumin-GdDTPA: albumin-gadolinium-diethylenetriaminepentaacetic acid

CAFs: cancer associated fibroblasts

Coll: collagen 1

COX-2: cyclooxygenase-2

ECM: extracellular matrix

EIA: enzyme immunoassay

FOV: field of view

GAPDH: glyceraldehyde 3-phosphate dehydrogenase

H\&E: hematoxylin and eosin

HMFs: human mammary fibroblasts

HRP: horseradish peroxidase

kDA: kilodalton

MRI: magnetic resonance imaging

PBS: phosphate buffered saline

$\mathrm{PGE}_{2}$ : prostaglandin E2

PS: permeability surface area product

SCID: severe combined immunodeficient

SDS-Page: sodium dodecyl sulfate poly-acrylamide

gel electrophoresis

SHG: second harmonic generation

shRNA: short hairpin RNA

siRNA: small interfering RNA

SMA: smooth muscle actin

TNBC: triple negative breast cancer

TPA: 12-O-tetradecanoylphorbol-13-acetate

VEGF: vascular endothelial growth factor

\section{ACKNOWLEDGMENTS}

We gratefully acknowledge useful discussions with Professor Michal Neeman and her colleagues at the Weizmann Institute regarding fibroblast immunostaining. We thank Dr. Dmitri Artemov for expert assistance. We thank Mr. Gary Cromwell for maintaining the cells and inoculating the tumors.

\section{CONFLICTS OF INTEREST}

None.

\section{FUNDING}

This work was supported by NIH R01CA82337, P50CA103175, R01CA136576, R01CA138515, R01CA73850, and P30CA006973.

\section{Author's contributions}

BK, IS, SK, MFP, DJ, MS, AP and ZMB participated in the experimental design, imaging, and analysis of 
the data. DJ, FW, and YM participated in the animal experiments. BK and ZMB drafted the manuscript. All authors contributed to the final editing of the manuscript.

\section{REFERENCES}

1. Smith WL, DeWitt DL and Garavito RM. Cyclooxygenases: structural, cellular, and molecular biology. Annu Rev Biochem. 2000; 69:145-182.

2. Xu L, Zhang L, Yi Y, Kang HK and Datta SK. Human lupus $\mathrm{T}$ cells resist inactivation and escape death by upregulating COX-2. Nat Med. 2004; 10:411-415.

3. Wang D and Dubois RN. Eicosanoids and cancer. Nature reviews Cancer. 2010; 10:181-193.

4. Thiel A, Mrena J and Ristimaki A. Cyclooxygenase-2 and gastric cancer. Cancer metastasis reviews. 2011; 30:387-395

5. Krysan K, Reckamp KL, Sharma S and Dubinett SM. The potential and rationale for COX-2 inhibitors in lung cancer. Anticancer Agents Med Chem. 2006; 6:209-220.

6. Howe LR. Inflammation and breast cancer. Cyclooxygenase/ prostaglandin signaling and breast cancer. Breast Cancer Res. 2007; 9:210

7. Singh-Ranger G, Salhab M and Mokbel K. The role of cyclooxygenase- 2 in breast cancer: review. Breast Cancer Res Treat. 2008; 109:189-198.

8. Koehne $\mathrm{CH}$ and Dubois RN. COX-2 inhibition and colorectal cancer. Semin Oncol. 2004; 31:12-21.

9. Mukherjee D, Nissen SE and Topol EJ. Risk of cardiovascular events associated with selective COX-2 inhibitors. JAMA. 2001; 286:954-959.

10. Suleyman H, Demircan B and Karagoz Y. Antiinflammatory and side effects of cyclooxygenase inhibitors. Pharmacological reports. 2007; 59:247-258.

11. Sinicrope FA and Gill S. Role of cyclooxygenase-2 in colorectal cancer. Cancer metastasis reviews. 2004; 23:63-75.

12. Sandler AB and Dubinett SM. COX-2 inhibition and lung cancer. Semin Oncol. 2004; 31:45-52.

13. Dohadwala M, Luo J, Zhu L, Lin Y, Dougherty GJ, Sharma S, Huang M, Pold M, Batra RK and Dubinett SM. Nonsmall cell lung cancer cyclooxygenase-2-dependent invasion is mediated by CD44. The Journal of biological chemistry. 2001; 276:20809-20812.

14. Chan MW, Wong CY, Cheng AS, Chan VY, Chan KK, To $\mathrm{KF}$, Chan FK, Sung JJ and Leung WK. Targeted inhibition of COX-2 expression by RNA interference suppresses tumor growth and potentiates chemosensitivity to cisplatin in human gastric cancer cells. Oncology reports. 2007; 18:1557-1562.

15. Coussens LM and Werb Z. Inflammation and cancer. Nature. 2002; 420:860-867.
16. Bocca C, Bozzo F, Bassignana A and Miglietta A. Antiproliferative effects of COX-2 inhibitor celecoxib on human breast cancer cell lines. Molecular and cellular biochemistry. 2011; 350:59-70.

17. Stasinopoulos I, O'Brien DR, Wildes F, Glunde $\mathrm{K}$ and Bhujwalla ZM. Silencing of cyclooxygenase-2 inhibits metastasis and delays tumor onset of poorly differentiated metastatic breast cancer cells. Mol Cancer Res. 2007; 5:435-442

18. Mosalpuria K, Hall C, Krishnamurthy S, Lodhi A, Hallman DM, Baraniuk MS, Bhattacharyya A and Lucci A. Cyclooxygenase-2 expression in non-metastatic triplenegative breast cancer patients. Molecular and clinical oncology. 2014; 2:845-850.

19. Stasinopoulos I, Mori N and Bhujwalla ZM. The malignant phenotype of breast cancer cells is reduced by COX-2 silencing. Neoplasia. 2008; 10:1163-1169.

20. Menter DG and Dubois RN. Prostaglandins in cancer cell adhesion, migration, and invasion. International journal of cell biology. 2012; 2012:723419.

21. Esbona K, Inman D, Saha S, Jeffery J, Schedin P, Wilke $\mathrm{L}$ and Keely P. COX-2 modulates mammary tumor progression in response to collagen density. Breast Cancer Res. 2016; 18:35.

22. Lyons TR, O'Brien J, Borges VF, Conklin MW, Keely PJ, Eliceiri KW, Marusyk A, Tan AC and Schedin P. Postpartum mammary gland involution drives progression of ductal carcinoma in situ through collagen and COX-2. Nat Med. 2011; 17:1109-1115.

23. Schedin P and Keely PJ. Mammary gland ECM remodeling, stiffness, and mechanosignaling in normal development and tumor progression. Cold Spring Harbor perspectives in biology. 2011; 3:a003228.

24. Provenzano PP, Inman DR, Eliceiri KW, Knittel JG, Yan L, Rueden CT, White JG and Keely PJ. Collagen density promotes mammary tumor initiation and progression. BMC medicine. 2008; 6:11.

25. Kakkad SM, Solaiyappan M, Argani P, Sukumar S, Jacobs LK, Leibfritz D, Bhujwalla ZM and Glunde K. Collagen I fiber density increases in lymph node positive breast cancers: pilot study. Journal of biomedical optics. 2012; 17:116017

26. Sabeh F, Ota I, Holmbeck K, Birkedal-Hansen H, Soloway P, Balbin M, Lopez-Otin C, Shapiro S, Inada M, Krane S, Allen E, Chung D and Weiss SJ. Tumor cell traffic through the extracellular matrix is controlled by the membraneanchored collagenase MT1-MMP. The Journal of cell biology. 2004; 167:769-781.

27. Byun JS and Gardner K. Wounds that will not heal: pervasive cellular reprogramming in cancer. Am J Pathol. 2013; 182:1055-1064.

28. Husarek KE, Zhang X, McCallinhart PE, Lucchesi PA and Trask AJ. Isolation of Murine Coronary Vascular Smooth Muscle Cells. Journal of visualized experiments. 2016. 
29. Salvado MD, Alfranca A, Haeggstrom JZ and Redondo JM. Prostanoids in tumor angiogenesis: therapeutic intervention beyond COX-2. Trends in molecular medicine. 2012; 18:233-243.

30. Zhang $\mathrm{Y}$ and Daaka Y. PGE2 promotes angiogenesis through EP4 and PKA Cgamma pathway. Blood. 2011; 118:5355-5364.

31. Chang SH, Liu CH, Conway R, Han DK, Nithipatikom $\mathrm{K}$, Trifan OC, Lane TF and Hla T. Role of prostaglandin E2-dependent angiogenic switch in cyclooxygenase 2 -induced breast cancer progression. Proceedings of the National Academy of Sciences of the United States of America. 2004; 101:591-596.

32. Muraki C, Ohga N, Hida Y, Nishihara H, Kato Y, Tsuchiya K, Matsuda K, Totsuka Y, Shindoh M and Hida $\mathrm{K}$. Cyclooxygenase-2 inhibition causes antiangiogenic effects on tumor endothelial and vascular progenitor cells. International journal of cancer. 2012; 130:59-70.

33. Katoh H, Hosono K, Ito Y, Suzuki T, Ogawa Y, Kubo H, Kamata H, Mishima T, Tamaki H, Sakagami H, Sugimoto Y, Narumiya S, Watanabe M and Majima M. COX-2 and prostaglandin EP3/EP4 signaling regulate the tumor stromal proangiogenic microenvironment via CXCL12-CXCR4 chemokine systems. Am J Pathol. 2010; 176:1469-1483.

34. Ristimaki A, Sivula A, Lundin J, Lundin M, Salminen T, Haglund C, Joensuu $\mathrm{H}$ and Isola J. Prognostic significance of elevated cyclooxygenase-2 expression in breast cancer. Cancer research. 2002; 62:632-635.

35. Davies G, Salter J, Hills M, Martin LA, Sacks N and Dowsett M. Correlation between cyclooxygenase-2 expression and angiogenesis in human breast cancer. Clinical cancer research. 2003; 9:2651-2656.

36. Wolf K, Mazo I, Leung H, Engelke K, von Andrian UH, Deryugina EI, Strongin AY, Brocker EB and Friedl P. Compensation mechanism in tumor cell migration: mesenchymal-amoeboid transition after blocking of pericellular proteolysis. The Journal of cell biology. 2003; 160:267-277.

37. Kakkad SM, Penet MF, Akhbardeh A, Pathak AP, Solaiyappan M, Raman V, Leibfritz D, Glunde K and Bhujwalla ZM. Hypoxic tumor environments exhibit disrupted collagen I fibers and low macromolecular transport. PloS one. 2013; 8:e81869.

38. Cirri P and Chiarugi P. Cancer associated fibroblasts: the dark side of the coin. Am J Cancer Res. 2011; 1:482-497.
39. Mao Y, Keller ET, Garfield DH, Shen K and Wang J. Stromal cells in tumor microenvironment and breast cancer. Cancer metastasis reviews. 2013; 32:303-315.

40. Orimo A, Gupta PB, Sgroi DC, Arenzana-Seisdedos F, Delaunay T, Naeem R, Carey VJ, Richardson AL and Weinberg RA. Stromal fibroblasts present in invasive human breast carcinomas promote tumor growth and angiogenesis through elevated SDF-1/CXCL12 secretion. Cell. 2005; 121:335-348.

41. Takai K, Le A, Weaver VM and Werb Z. Targeting the cancer-associated fibroblasts as a treatment in triplenegative breast cancer. Oncotarget. 2016; 7: 82889-82901. doi: 10.18632/oncotarget.12658.

42. Fordyce CA, Patten KT, Fessenden TB, DeFilippis R, Hwang ES, Zhao J and Tlsty TD. Cell-extrinsic consequences of epithelial stress: activation of protumorigenic tissue phenotypes. Breast Cancer Res. 2012; $14: R 155$.

43. Riquet FB, Lai WF, Birkhead JR, Suen LF, Karsenty G and Goldring MB. Suppression of type I collagen gene expression by prostaglandins in fibroblasts is mediated at the transcriptional level. Molecular medicine. 2000; 6:705-719.

44. Martinez-Outschoorn UE, Curry JM, Ko YH, Lin Z, Tuluc M, Cognetti D, Birbe RC, Pribitkin E, Bombonati A, Pestell RG, Howell A, Sotgia F and Lisanti MP. Oncogenes and inflammation rewire host energy metabolism in the tumor microenvironment: RAS and NFkappaB target stromal MCT4. Cell Cycle. 2013; 12:2580-2597.

45. Watwe V, Javle M, Lawrence D, Groth J, Iyer R, El-Hajjar $\mathrm{D}$ and Geradts J. Cyclooxygenase-2 (COX-2) levels before and after chemotherapy: a study in rectal cancer. Am J Clin Oncol. 2005; 28:560-564.

46. Pathak AP, Artemov D, Ward BD, Jackson DG, Neeman $\mathrm{M}$ and Bhujwalla ZM. Characterizing extravascular fluid transport of macromolecules in the tumor interstitium by magnetic resonance imaging. Cancer research. 2005; 65:1425-1432.

47. Kakkad SM, Solaiyappan M, O'Rourke B, Stasinopoulos I, Ackerstaff E, Raman V, Bhujwalla ZM and Glunde K. Hypoxic Tumor Microenvironments Reduce Collagen I Fiber Density. Neoplasia. 2010; 12:608-617.

48. Heo SC, Lee KO, Shin SH, Kwon YW, Kim YM, Lee CH, Kim YD, Lee MK, Yoon MS and Kim JH. Periostin mediates human adipose tissue-derived mesenchymal stem cellstimulated tumor growth in a xenograft lung adenocarcinoma model. Biochimica et biophysica acta. 2011; 1813:2061-2070. 